\title{
PAPER
}

\section{Heat conduction in a hard disc system with non-conserved momentum}

To cite this article: P L Garrido and Joel L Lebowitz J. Stat. Mech. (2020) 053205

View the article online for updates and enhancements.

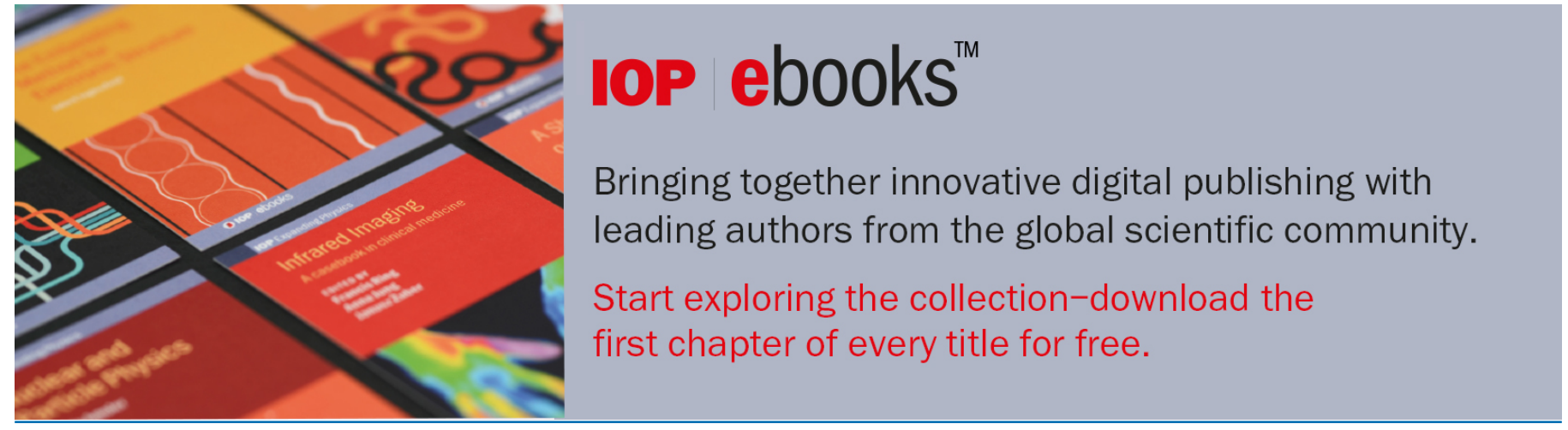

This content was downloaded from IP address 150.214 .103 .147 on $27 / 09 / 2021$ at $11: 14$ 


\title{
Heat conduction in a hard disc system with non-conserved momentum
}

\author{
P L Garrido ${ }^{1,3}$ and Joel L Lebowitz ${ }^{2}$ \\ ${ }^{1}$ Instituto Carlos I de Física Teórica y Computacional, Universidad de \\ Granada, E-18071 Granada, Spain \\ 2 Departments of Mathematics and Physics, Rutgers University, \\ Piscataway, NJ 08854, United States of America \\ E-mail: garrido@onsager.ugr.es and lebowitz@math.rutgers.edu
}

Received 14 November 2019

Accepted for publication 17 February 2020

Published 22 May 2020

Online at stacks.iop.org/JSTAT/2020/053205

https://doi.org/10.1088/1742-5468/ab7af5

\begin{abstract}
We describe results of computer simulations of steady state heat transport in a fluid of hard discs undergoing both elastic interparticle collisions and 'pseudo collisions' which do not conserve momentum. The latter are done by picking particles at random and randomizing the directions of their velocities. The system consists of $N$ discs of radius $r$ in a unit square, periodic in the $y$-direction and having thermal walls with different temperatures at $x=0$ and at $x=1$. We extrapolate results from different $N$, to $N \rightarrow \infty, r \rightarrow 0$, such that $\pi r^{2} N=1 / 2$. We find that in the (extrapolated) hydrodynamic limit $N \rightarrow \infty$, the systems' local density and temperature profiles are those of local thermodynamic equilibrium (LTE), the corresponding pressure is constant independent of position and the heat flux obeys Fourier's law. The variance of global quantities, such as the total energy, deviates from its local equilibrium value in a form consistent with macroscopic fluctuation theory.
\end{abstract}

Keywords: molecular dynamics, numerical simulations, stationary states, transport processes/heat transfer

\footnotetext{
${ }^{3}$ Author to whom any correspondence should be addressed.
} 


\section{Contents}

1. Introduction 2

2. Local equilibrium 4

3. Temperature profiles and Fourier's law 6

4. Fluctuations $\quad 8$

5. Concluding remarks $\quad 9$

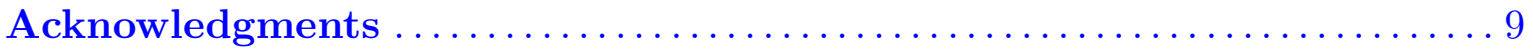

$\begin{array}{ll}\text { Appendix } & 9\end{array}$

$\begin{array}{ll}\text { References } & 12\end{array}$

\section{Introduction}

We continue our investigation, via molecular dynamics (MD), of the nonequilibrium stationary states of a system of $N$ hard discs of radius $r$ in a unit square [1]. The system has areal density $\rho=N \pi r^{2}=0.5$, periodic boundary conditions in the $y$-direction and thermal walls at $x=0$ and $x=1$ with temperatures $T_{0}$ and $T_{1}(=1)$ respectively for all computer simulated cases in this paper. We have simulated different $N$ values ranging from 460 up to 5935 (see appendix for all the technical details about the computer simulation) in order to do a finite size analysis and obtain the hydrodynamic description of the system, in the limit, $N \rightarrow \infty, r \rightarrow 0$.

The dynamics of the discs consists of linear displacements at constant velocity and elastic collisions when two discs meet. Additionally the dynamics has a part that breaks the bulk momentum conservation of the system dynamics. We introduced such a mechanism recently in the context of kinetic equations for the one particle distribution, $f(r, v, t)[2]$. There we added to the usual collision term, $Q_{\mathrm{c}}(f)$, such as Boltzmann, Boltzmann-Enskog and BGK, a linear collision term, $Q_{\mathrm{D}}(f)$, which randomizes velocities but conserves energy. We multiplied this term by a parameter $\alpha$, leading to a kinetic equation for $f(r, v, t)$,

$$
\partial_{t} f+v \cdot \nabla f=Q_{\mathrm{C}}(f)+\alpha Q_{\mathrm{D}}(f)
$$

This led to an evolution of $f$ which had only two conservation laws, particle and energy density, i.e. no momentum conservation. $Q_{\mathrm{D}}(f)$ represents particle collisions with fixed obstacles, as in a Lorentz gas. It corresponds physically to a fluid moving in a porous medium [3]. Diffusively scaling space and time [4] enabled us to derive rigorously, from the modified kinetic equations, macroscopic equations for the two conserved quantities 
[5]. We are not aware of such rigorous derivation for the full conservation laws including momentum.

In this note we describe MD simulations of hard discs with a dynamics which destroys momentum conservation in a different way from that modeled in reference [2] but has physically a similar effect. We do not assume here the validity of any kinetic equation for $f$. The momentum destroying dynamics is done as follows: following each elastic collision between a pair of particles in the system we pick $\gamma$ particles at random from the remaining $(N-2)$ particles and randomize the direction of their velocities. We do not consider the particles involved in the actual collision to exclude dynamic pathologies. We have simulated $\gamma=0,0.3,1,5$ and $10 . \gamma=0$ corresponds to purely dynamical evolution as in reference [1] while for $\gamma=0.3$ we wait for ten interparticle collisions before choosing three particles at random.

In addition to these bulk collisions there are also collisions with the thermal walls. When a disc hits a thermal wall it gets a new normal component of the velocity with respect to the boundary. Its value is obtained from a Maxwellian velocity distribution with the temperature that corresponds to the wall. We use this combined dynamics to the study of the stationary state. This was previously investigated for the case $\gamma=0$ [1]. We have done simulations for $T_{0}=1,3,5,7, \ldots, 21$.

We find that for $N \rightarrow \infty$ our system satisfies locally the equilibrium equation of state:

$$
P \pi r^{2} \equiv Q=T(x) \rho(x) H(\rho(x))
$$

where $\rho(x), T(x)$ are the local areal density and the local temperature respectively and $P$ is the pressure. $H$ is independent of $\gamma$ and is given by its equilibrium value, see (4).

In addition we observe that the heat current follows Fourier's law

$$
J_{\mathrm{H}} / r \equiv J=-\kappa(T, \rho ; \gamma) \frac{\mathrm{d} T}{\mathrm{~d} x} \quad x \in[0,1]
$$

where $J_{\mathrm{H}}$ is the heat current. $P$ and $J_{\mathrm{H}}$ are also observed to be independent of $x$ in the stationary state. This follows from the uniformity of the system in the $y$-direction.

The expressions for $H(\rho)$ and $\kappa(T, \rho ; \gamma)$ obtained numerically will be compared with some theoretical approximations. For instance, $H(\rho)$ proposed by Henderson [6] is known to be a very good approximation for $\rho \lesssim 0.6$ :

$$
H(\rho)=\frac{1+\rho^{2} / 8}{(1-\rho)^{2}}-0.043 \frac{\rho^{4}}{(1-\rho)^{3}}
$$

Observe that based on computer simulations and some theoretical analysis, the 2D system of hard discs at equilibrium is believed to have a phase transition at $\rho \simeq 0.7[7]$.

For $\kappa(T, \rho ; \gamma)$ we first note that to the extent that $(3)$ holds, i.e. $J$ is a linear functional of the local gradient, $J$ will be proportional to $\sqrt{T}$. Moreover, it will vanish as the distance between particles and the time between collisions goes to zero as $r \rightarrow 0$ so it has to be rescaled by $r$ as is done in (3). Thus, in the limit $N \rightarrow \infty$ 


$$
\kappa(T, \rho ; \gamma)=\sqrt{T} K(\rho ; \gamma)
$$

For $K(\rho ; \gamma)$ we use an expression for the conductivity derived in reference [2] from an approximate solution of the Enskog equation with a momentum destroying collision term proportional to $\alpha$ : see equation (78) in [2]. We replace the dimensionless parameter $\alpha_{s}$ there by a function $\phi(\gamma)$. This gives

$$
K(\rho ; \gamma)=\frac{1+(3+16 \phi(\gamma) \rho) \rho \chi(\rho)+(19+9 \pi)(\rho \chi(\rho))^{2} / 4 \pi}{\sqrt{\pi}(\chi(\rho)+4 \pi \phi(\gamma))}
$$

where $\chi(\rho)=(H(\rho)-1) / 2 \rho . \phi(\gamma)$ is chosen to give accurate results at low densities. This gives, for the values of $\gamma$ simulated here, $\phi(0.3)=0.09, \phi(1)=0.286, \phi(5)=1.685$ and $\phi(10)=5.50$.

We solve equations (2) and (3) using $H$ and $K$ from equations (4) and (6) respectively with the boundary conditions

$$
T(0)=T_{0}, \quad T(1)=1, \quad \bar{\rho}=\int_{0}^{1} \mathrm{~d} x \rho(x)=0.5
$$

to obtain expressions for $\rho(x)$ and $T(x), J$ and $P$. We check these against the results of the simulations in sections 2 and 3 . In section 4 we consider $1 / N$ deviations from local equilibrium as seen in the energy fluctuations and find that they agree with the macroscopic fluctuating theory.

\section{Local equilibrium}

The local equilibrium hypothesis used in the last section assumes that in the hydrodynamic description of a macroscopic system we can locally define equilibrium thermodynamic observables that obey the equilibrium relations between them. To check if the equilibrium equation of state (EOS) holds in each stripe parallel to the $y$-axis (see appendix) we define the local density for the stripe $s, \rho(s)$ as the number of particle centers in the stripe:

$$
\rho(s)=\frac{1}{N} \sum_{i: r_{i} \in B(s)} 1
$$

where $B(s)$ is the set of points belonging to the stripe $s$ (see appendix). We then use the virial theorem to compute the local pressure $Q_{\mathrm{v}}(s)$ :

$$
Q_{\mathrm{v}}(s)=\rho(s) T(s)+\frac{1}{2 \Delta_{B}^{2} \tau} \sum_{n: t_{n} \in[0, \tau] ; r_{i}(t) \in B(s)} r_{i j}\left(t_{n}\right) \cdot p_{i j}\left(t_{n}\right)
$$

where $r_{i j}=r_{i}-r_{j}, p_{i j}=p_{i}-p_{j}, \Delta_{B}$ is the width of a stripe, and the sum runs over all particle-particle collisions that occur in the cell $s$ in the time interval $[0, \tau]$ letting $\tau$ be large enough for the right-hand side of equation (9) to be independent of $\tau$. 
Heat conduction in a hard disc system with non-conserved momentum

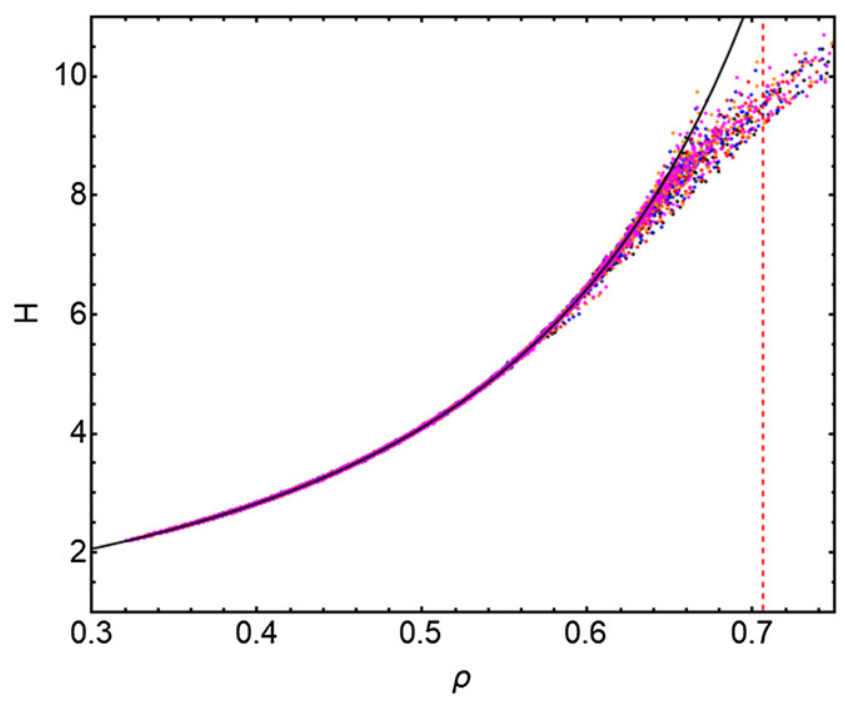

Figure 1. $H(\rho(s))=Q_{v}(s) / \rho(s) T(s)$ vs $\rho(s)$ for $s=4, \ldots, N_{\mathrm{B}}-3$ for all the simulated $N$ 's in table A1 and $\Delta T=T_{0}-T_{1}=0,2, \ldots, 20$. Black, red, blue, orange and magenta points are for $\gamma=0,0.3,1,5$ and 10 , respectively. The dotted vertical line is the observed equilibrium phase transition critical value $(\gamma=0$ and $\Delta T=0): \rho_{\mathrm{c}} \simeq 0.7062$. The black solid line is the $H(\rho)$ function corresponding to the Henderson's equation of state (see text).

First we checked that the virial pressure $Q_{v}$ is constant all over the system for each $N$, temperature gradient and $\gamma$ 's and we got the average over the stripes. Finally from such data we extrapolated its value to $N \rightarrow \infty$. We also checked that this value agrees with the pressure measured at the thermal walls, by computing the momentum transfer to the wall.

In order to check the local equilibrium hypothesis we plot $H(\rho(s))=Q_{v}(s) / \rho(s) T(s)$ vs $\rho(s)$ for all the stripes $s$ (except the ones near the heat bath boundaries) and for all the simulations done: $\gamma$ 's, $\Delta T$ 's and $N$ 's. In total there are 17195 data points represented in figure 1 .We observe in figure 1 that most of the points with local densities $\lesssim 0.6$ follow the same curve independently of the position of the stripe, the number of particles $N$, the temperature gradient $\Delta T$ or the randomization intensity $\gamma$. We observe that Henderson's EOS (4) follows the data almost perfectly for low densities and only deviates when approaching the expected equlibrium phase transition critical density at $\rho_{\mathrm{c}} \simeq 0.7062[7]$. Moreover, the data lose the scaling property for densities larger than 0.6. We believe that is due mainly to the small size of the virtual stripes when the particles begin to crystallize in a hexagonal lattice and the center of the particles tend to be ordered and aligned [8]. Therefore nearby stripes may contain one or two lines of centers affecting microscopically the values of the measured densities. Finally we conclude from the data analysis that our nonequilibrium system has the local equilibrium property in the range of densities $[0,0.6]$.

The quality of the data allows us to look in detail at the quality of the Henderson EOS. We observe that for $\rho \leqslant 0.6$ the Henderson EOS has less than $1 \%$ relative error with the data. 


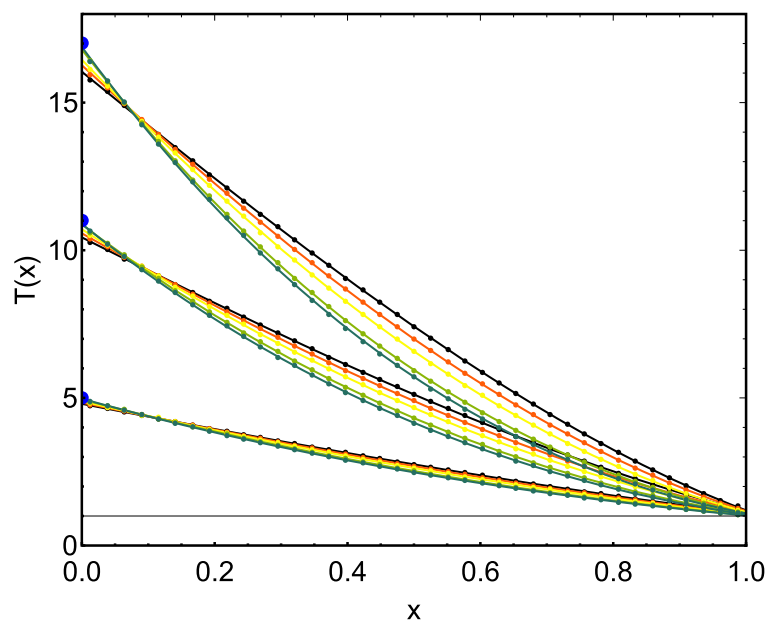

Figure 2. Thermal profiles for $N=4000, \Delta T=4,10,16$ and $\gamma=0$ (dark green), $\gamma=0.3$ (green), $\gamma=1$ (yellow), $\gamma=5$ (red) and $\gamma=10$ (black). Solid lines are the corresponding fitted functions. Big points at $x=0$ show the simulated $T_{0}$ values.

\section{Temperature profiles and Fourier's law}

The local temperature is defined as the average local kinetic energy per particle at each stripe. That is,

$$
T(s)=\frac{1}{2 \rho(s) N M} \sum_{t=1}^{M} \sum_{i: r_{i}(t) \in B(s)} v_{i}(t)^{2}
$$

where $M$ is the number of measurements $\left(M=10^{5}\right)$. We assume that a particle belongs to a stripe if its center is in the stripe. This computational method is efficient but it does not compute correctly the density behavior near the walls.

As an example, we show in figure 2 the temperature profiles for $N=4000, \Delta T=4$, 10 and 16 and different $\gamma$ values. We see that all the measured profiles are monotone decreasing functions with positive curvature. We also see that the size effects are larger as we increase the temperature gradient for a given $\gamma$ value.

We observe that the extrapolated profiles up to the boundaries do not coincide with the temperature values used in the simulations. This phenomena is known as thermal resistance. Kinetic theory arguments predict that this temperature gap goes to zero as the mean free path which behaves like $N^{-1 / 2}$ when $N \rightarrow \infty$. We have checked this prediction by fitting the data for each temperature profile to a polynomial with positive curvature. We extrapolate the fitted functions to the boundary points $x=0$ and $x=1$ getting $T_{0}^{\mathrm{e}}$ and $T_{1}^{\mathrm{e}}$ respectively. We define the relative gap:

$$
G_{i}(N)=\frac{\left(T_{i}-T_{i}^{e}(N)\right)}{T_{i}} \simeq \frac{G_{i}}{\sqrt{N}} \quad(i=0,1)
$$

We got the values of $G_{i}$ versus $\Delta T$ by extrapolating $G_{0,1}(N) \sqrt{N}$ to $N \rightarrow \infty$ for the different values of $\gamma$. We confirm the behavior with $N^{-1 / 2}$ of $G_{i}(N)$. We see that for a 


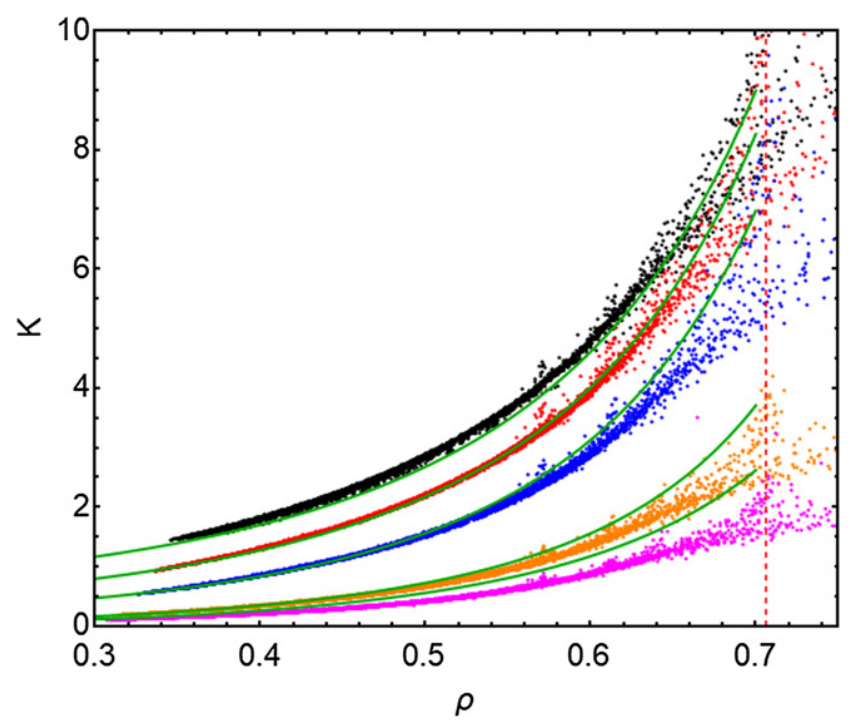

Figure 3. $K(s)$ vs $\rho(s)$ for the virtual boxes $s=2, \ldots, N_{\mathrm{B}}-1$ for all the simulated $N$ 's in table A1 and $\Delta T=T_{0}-T_{1}=0,2, \ldots, 20$. Black, red, blue, orange and magenta dots are for $\gamma=0,0.3,1,5$ and 10 respectively. The red vertical line is the observed equilibrium phase transition critical value when $\gamma=0$ and $\Delta T=0$ : $\rho_{\mathrm{c}} \simeq 0.7062$. The solid lines are obtained by fitting the parameters $\phi(\gamma)$ in the Boltzmann-Enskog expression for the thermal conductivity [see equation (6)] to the data and by using the Henderson EOS.

given temperature gradient and size $N$ the gap increases with $\gamma$. On the other hand, for any $\gamma$ value, the thermal resistance increases with the gradient.

To check the validity of Fourier's law (3) we computed at each stripe

$$
K(\rho ; \gamma)=-\frac{J}{\sqrt{T(x)}}\left(\left.\frac{\mathrm{d} T}{\mathrm{~d} x}\right|_{x=x(\rho)}\right)^{-1}
$$

as a function of $\rho$. If Fourier's law holds $K(\rho ; \gamma)$ should be, for each $\gamma$, a universal curve independent of the parameters that define the stationary state: $T_{0}, T_{1}$ and $\bar{\rho}$ and the $x$ used. The derivative of the thermal profile is analytically done over the fitted profile. The use of fitting functions with positive curvature reduces the dispersion of the values of the derivatives due to the typical 'waves' around the average profile that one obtains when using an arbitrary polynomial. For the thermal conductivity analysis we have discarded the points near the thermal walls to minimize the boundary effects.

We draw figure 3 by computing the derivative of the fitted function at the center of each virtual box, associating to the point the measured average density in the box. We see there that the data follows quite well a unique curve for each given $\gamma$ value. Let us stress that for each $\gamma$ we are plotting around 3600 data points corresponding to systems with different thermal gradients and number of particles in each of the stripes where local variables are defined. Observe that the $N$ dependence is not visible and that the $K$ decreases with $\gamma$ as expected. We observe deviations to the unique curve when approaching the critical density. As we see, we have obtained a reasonable description 
Heat conduction in a hard disc system with non-conserved momentum
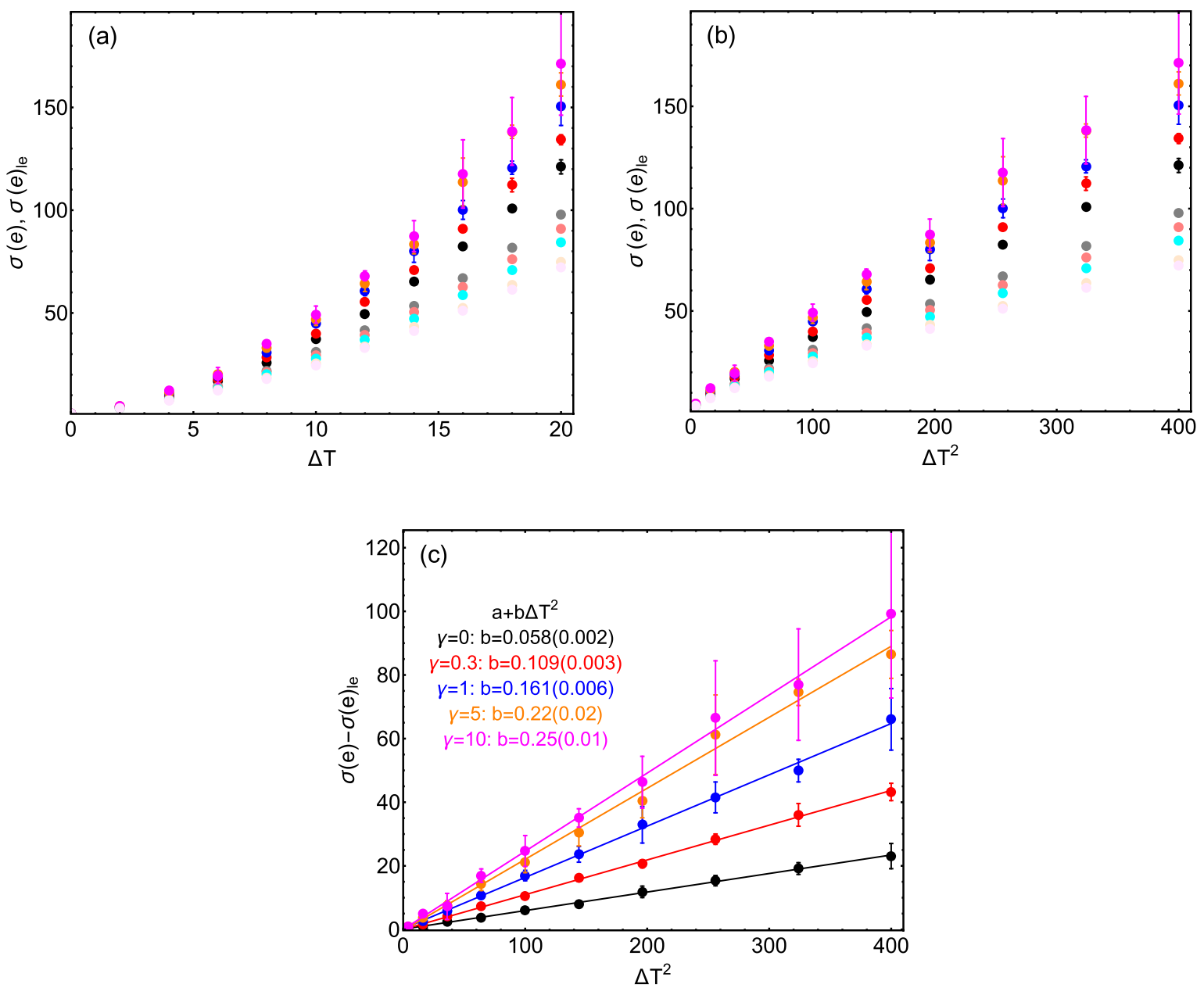

Figure 4. (a) Energy fluctuations $\sigma(e)$ vs $\Delta T$ for $\gamma=0$ (black points), 0.3 (red points), 1 (blue points), 5 (orange points) and 10 (magenta points). We also plot the energy fluctuations assuming local equilibrium, $\sigma(e)_{\text {le }}$, for $\gamma=0$ (gray points), $\gamma=0.3$ (pink points), $\gamma=1$ (cyan points), $\gamma=5$ (light orange points) and $\gamma=10$ (light magenta points). (b) Same data as left figure but plotted versus $\Delta T^{2}$ to see the nontrivial curvature of the data sets. (c) Difference between the measured energy fluctuations and the local equilibrium energy fluctuations. Solid lines are linear fits to the data.

by $K(\rho)$ of equation $(6)$ for $\rho \leqslant 0.6$. Again the deviations from the theory to the data increase with $\gamma$.

\section{Fluctuations}

We measured the fluctuations of the energy per particle in the stationary state:

$$
m\left(e_{N}\right)=\left\langle e_{N}^{2}\right\rangle-\left\langle e_{N}\right\rangle^{2}, \quad e_{N}=\frac{1}{N} \sum_{i=1}^{N} e\left(v_{i}\right)
$$


this has the asymptotic behavior:

$$
\sigma(e)=\lim _{N \rightarrow \infty} N m\left(e_{N}\right)
$$

plotted in figure $4(\mathrm{a})$.

It is well known, for certain exactly solvable models, e.g. SEP, KMP that even when the system is in LTE in the macroscopic limit $(N \rightarrow \infty)$ the fluctuation in global quantities deviates from their LTE values [9]. For our system

$$
\sigma_{\mathrm{le}}(e)=\rho^{-1} \int_{0}^{1} \mathrm{~d} x \rho(x) T(x)^{2}
$$

This is plotted in figure $4(\mathrm{~b})$.

In figure $4(\mathrm{c})$ we show $\delta(e) \equiv \sigma(e)-\sigma_{\text {le }}(e)$ and observe that $\delta(e)=b(\gamma)(\Delta T)^{2}$ with $b(\gamma)>0$ monotone increasing with $\gamma: b(0)=0.058(0.002), b(0.3)=0.109(0.003)$, $b(1)=0.161(0.006), b(5)=0.22(0.02)$ and $b(10)=0.25(0.01)$. This is of the same form as that founded for the exactly solvable models. It is also of the form found by macroscopic fluctuation theory (MFT) [10]. The coefficient of $(\Delta T)^{2}$ can be related in MFT (when there is only a single macroscopic variable) to the compressibility and transport coefficients. It can be positive or negative. We have not tried to compute $b(\gamma)$ for our system but it is noteworthy that the linear $(\Delta T)^{2}$ dependence holds also for deterministic systems with $\gamma=0$.

\section{Concluding remarks}

We have studied via MD simulations the NESS of a system of hard discs with an imposed nonuniform temperature in the hydrodynamic scaling limit, $r \rightarrow 0, N \pi r^{2}=0.5$. The dynamics conserve energy but not momentum. The results provide strong evidence for the system being in LTE. We also found evidence of long range correlations behaving as $N^{-1}$ giving rise to non LTE variances in global quantities.

\section{Acknowledgments}

We thank H Spohn, C Bernardin and specially R Esposito and D Gabrielli for very helpful correspondence. This work was supported in part by AFOSR (Grant FA-9550-161-0037). P L G was supported also by the Spanish Government Project FIS2017-84256-P funded by MINECO/AEI/FEDER. We thank the IAS System Biology divison for its hospitality during part of this work.

\section{Appendix}

Starting in an ordered initial configuration we let the system relax towards its stationary state during $5 \times 10^{4} \mathrm{~N}$ particle collisions and then we do measurements for each $10^{2} \mathrm{~N}$ 
Table A1. $N$ is the different number of particles in the bulk simulated in this paper. $N_{\mathrm{B}}$ is the number of virtual stripes in which we divide the system to measure local observables.

\begin{tabular}{lccc}
\hline$N$ & $N_{\mathrm{B}}$ & $N$ & $N_{\mathrm{B}}$ \\
\hline 460 & 13 & 3434 & 36 \\
941 & 19 & 3886 & 39 \\
1456 & 23 & 4367 & 41 \\
1927 & 27 & 4875 & 43 \\
2438 & 30 & 5412 & 46 \\
2900 & 33 & 5935 & 48 \\
\hline
\end{tabular}

particle collision obtaining $M=10^{5}$ data. The errors in all magnitudes are $3 \sigma$ with $\sigma$ being the standard deviation of the set of measurements. We have measured global magnitudes as the energy per particle, $e_{N}$, the pressure, $Q_{N}$ and the heat current $J_{N}$ that are defined for the simulations:

$$
\begin{aligned}
e_{N} & =\frac{1}{M} \sum_{t=1}^{M} \frac{1}{N} \sum_{i=1}^{N} e\left(v_{i}(t)\right) \\
Q_{N} & =\frac{\pi r^{2}}{\tau_{\text {col }}} \sum_{n=1}^{N_{\mathrm{wc}}}\left(\Delta v_{x}\right)_{n} \\
J_{N} & =\frac{r}{\tau_{\text {col }}} \sum_{n=1}^{N_{\mathrm{wc}}}(\Delta e(v))_{n}
\end{aligned}
$$

where

$$
e(v)=\frac{1}{2}\left(v_{i, x}^{2}+v_{i, y}^{2}\right)
$$

is the kinetic energy of particle $i . N_{\text {wc }}$ is the number of hard discs collisions with the walls in the time interval $\left[0, \tau_{\text {col }}\right]$ once the systems reaches the stationary state. $(\Delta A)_{n}$ is the variation of the magnitude $A$ before and after the $n$th collision with the wall: $A=v_{x}$ or $e(v)$.

In order to measure local observables such as the density, temperature and the virial pressure. We divide the system into virtual stripes parallel to the heat bath walls. We choose their width to be of order $4 r$. More precisely, the number of stripes is $N_{\mathrm{B}}=\operatorname{int}(1 / 2 r)$ and the stripes width $l=1 / N_{\mathrm{B}}$. Observe that $N_{\mathrm{B}}$ depends on the particle radius that also depends on $N$ as is seen in table A1.

For each averaged observable we find a systematic dependence on $N$ and in order to check the hydrodynamic equations we need to extrapolate to their limit, $N \rightarrow \infty$ value. For instance we show in figure A1 the pressure measured at the walls, $Q_{N}$, as an example of such systematic size dependence. We plot there $Q_{N}$ for $\gamma=1$. In order to extrapolate the data for a given $\Delta T$ and $\gamma$ to the $N \rightarrow \infty$ limit, it is typically enough to use a second order polynomial fitting function: $a_{N}=a+a_{1} / N+a_{2} / N^{2}$. In figure A1 we plot the fits 
Heat conduction in a hard disc system with non-conserved momentum

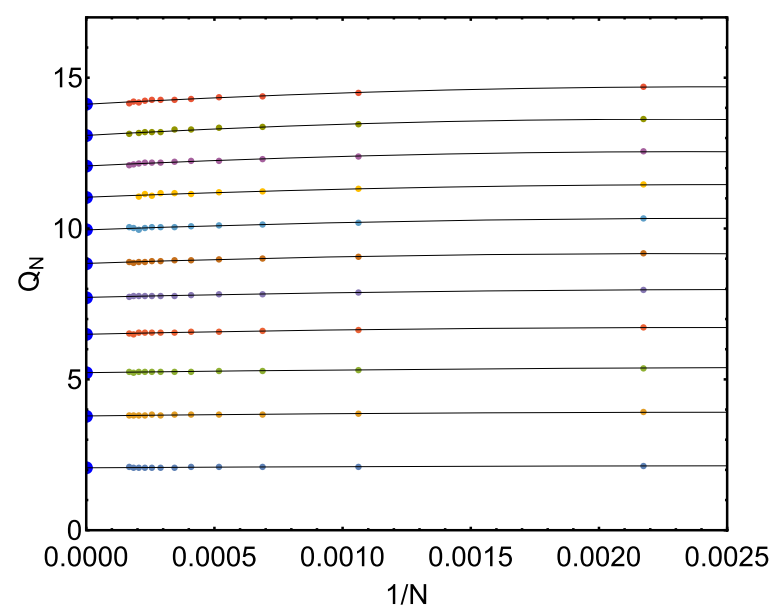

Figure A1. Pressure, $Q_{N}$, for $\gamma=1$ and $\Delta T=T_{0}-T_{1}=0,2, \ldots, 20$ (from bottom to top inside each graph) as a function of $1 / N$. The points on the $1 / N=0$ axis correpond to the extrapolation of a parabolic least square fit to the data (solid lines) for each $T_{0}$. Error bars are smaller than the points size.

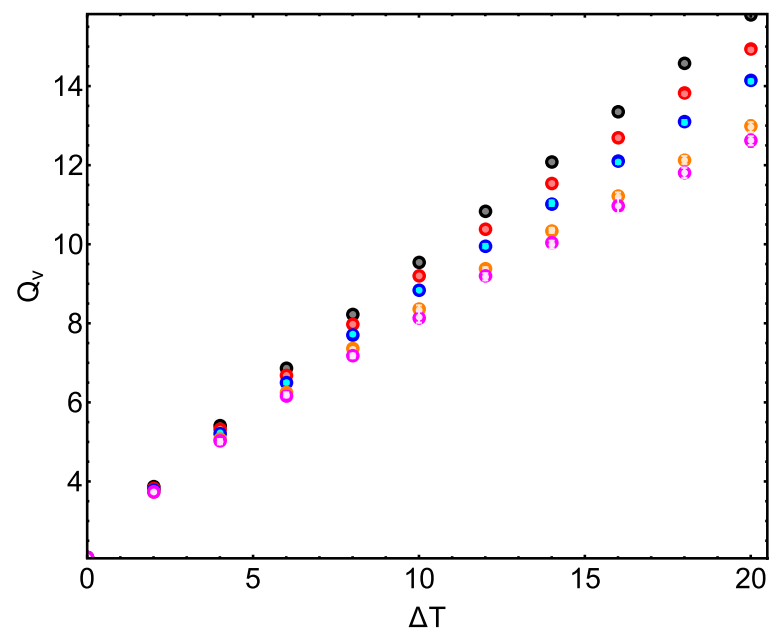

Figure A2. Extrapolation of the virial pressure, $Q_{\mathrm{v}}=\lim _{N \rightarrow \infty} Q_{v, N}$ and the pressure $Q=\lim _{N \rightarrow \infty} Q_{N}$ as a function of $\Delta T$. Big black, red, blue, orange and magenta points are for $Q_{\mathrm{v}}$ and $\gamma=0,0.3,1,5$ and 10 respectively. Small gray, pink, cyan, light orange and light magenta points are for $Q$ and $\gamma=0,0.3,1,5$ and 10, respectively.

as thin dashed lines. The big points in the figures are the extrapolated $Q$ 's from the fitted functions for each $\Delta T$ values. These limiting values are essential to get a coherent picture of the system's behavior. For instance, in the pressure case, we also measured the local pressure by using the virial expression. First we observe that the data at the boundaries are distorted by the way we have defined the density measurements. We discard such boundary data in the analysis. Second, we tried to fit several functions to the bulk data and we concluded that the best one is the constant function. The virial pressure is derived from classical mechanics and therefore its constant value along the 
stripes indicates a kind of mechanical equilibrium at the system stationary state. We again use the fitted values for different $N$ 's to extrapolate the data to $N \rightarrow \infty$ by using a second order polynomial in $1 / N$. Finally, we can compare these asymptotic values with the pressure $Q$ measured on the walls and we get a very good match.

We see in figure A2 the extrapolated $Q$ and $Q_{v}$. At a glance we do not see any systematic deviation. In fact the relative error $100\left|Q-Q_{v}\right| / Q$ runs between $0.01 \%$ and $0.4 \%$ which is very small and it indicates the good quality of the data obtained in the computer simulation.

\section{References}

[1] del Pozo J J, Garrido P L and Hurtado P I 2015 Scaling laws and bulk-boundary decoupling in heat flow Phys. Rev. E 91032116

del Pozo J J, Garrido P L and Hurtado P I 2015 Probing local equilibrium in nonequilibrium fluids Phys. Rev. E 92022117

[2] Garrido P L and Lebowitz J L 2018 Diffusion equations from kinetic models with non-conserved momentum Nonlinearity 3154

[3] Callen H B 1960 Thermodynamics (New York: Wiley) ch 17

[4] Spohn H 1991 Large Scale Dynamics of Interacting Particle Systems (Berlin: Springer)

[5] Esposito R, Garrido P L, Lebowitz J L and Marra R 2019 Diffusive limit for a Boltzmann-like equation with non-conserved momentum Nonlinearity $\mathbf{3 2} 4834$

[6] Henderson D 1975 A simple equation of state for hard discs Mol. Phys. 30971

Henderson D 1977 Monte Carlo and perturbation theory studies of the equation of state of the two-dimensional Lennard-Jones fluid Mol. Phys. 34301

[7] A Mulero (ed) 2008 Theory and Simulation of Hard-Sphere Fluids and Related Systems (Lect. Notes Phys. vol 753) (Berlin: Springer)

[8] Thorneywork A L, Abbott J L, Aarts D G A L and Dullens R P A 2017 Two-dimensional melting of colloidal hard spheres Phys. Rev. Lett. 118158001

[9] Derrida B, Lebowitz J L and Speer E R 2002 Large deviation of the density profile in the steady state of the open symmetric simple exclusion process J. Stat. Phys. 107599

Bertini L, Gabrielli D and Lebowitz J L 2005 Large deviations for a stochastic model of heat flow J. Stat. Phys. 121843

[10] Bertini L, de Sole A, Gabrielli D, Jona Lasinio G and Landim C 2015 Macroscopic fluctuation theory Rev. Mod. Phys. 87593 